\title{
Viscosity coefficients in nonideal liquid mixture
}

\author{
(C) Arnold Sh. Bicbulatov, ${ }^{1}$ and Asia A. Usmanova ${ }^{2+}$ \\ ${ }^{1}$ Department of Chemical Engineering Apparatus. Kazan National Research Technological University. \\ Siberian Ave., 68. Kazan, 420029. Tatarstan Republic. Russia. \\ Phone:+7 (843)231-40-32.E-mail: iraida@kstu.ru \\ ${ }^{2}$ Department of Automation Sistems and Prosess Control. Kazan National Research Technological \\ University. Karl Marx St., 72. Kazan, 420015. Tatarstan Republic. Russia. \\ Phone:+7 (843)231-42-72.E-mail: sautp@yandex.ru
}

\begin{abstract}
*Supervising author; ${ }^{+}$Corresponding author Keywords: viscosity coefficients, nonideal liquid mixtures, kinetic theory hart spheres model, thermodynamics of irreversible processes, activity coefficients.
\end{abstract}

\begin{abstract}
To calculate various heat and mass transfer processes, reliable data on the molecular momentum transfer coefficient are required, which should fit seamlessly in to the General algorithm for modeling and calculating various mass transfer processes and devices in the chemical and petrochemical industries in the form of unified programs. This approach is proposed in the description of the momentum transfer mechanism, which is implemented by generalizing the kinetic equations of the model of solid spheres of a system of dense media with the help of methods of the thermodynamics of irreversible processes for associated Prigogine solution model. This allows a more accurate description of the momentum transfer in nonideal solutions. Within the framework of this model, the expression of the collision therm in kinetic equations is refined, which significantly expands the boundaries of the theory's application. A method for calculation activity coefficient is developed based on experimental data in the liquid-vapor system and the Wilson the equation. This will allow us to improve quantitatively the description of the phenomena of momentum transfer. A comparision was made between the calculated and experimental viscosity coefficients for a highly nonideal aceton-water solution, in which the activity coefficient varies on both components from 1 to 5 units. The average discrepancy between the data at different concentrations is $30 \%$. At the sesame, the discrepancy between the coefficients, calculated according to the theory of solid spheres and experimental data reaches up to 10 times. When specifying the values of the activity coefficients and the interaction parameter between molecules the proposed method will improve the result.
\end{abstract}

\section{References}

[1] A.Sh. Bicbulatov, and A.A. Usmanova. Refined description of momentum transfer in real liquid mixtures. Butlerov Communications. 2020. Vol.64. No.11. P.154-158. DOI: 10.37952/ROI-jbc-01/20-64$11-154$

[2] A.S. Bikbulatov. Modified description of pulse transfer. Zhurn. Physical Chemistry. 1995. Vol.69. No.2. P.362-364. (russian)

[3] D. Ferziger, G. Kaper. Mathematical theory of the transfer process in gases. Moscow: World. 1976. 554p. (russian)

[4] M.K. Tham, K.E. Gubbins. Theory of Multicomponent Dense Fluid Mixtures of Rigid Spheres. Zhurn. Physical Chemistry. 1971. Vol.55. No.1. P.268-279.

[5] S. Katherihe, R.A. Howard., Mc. Alister. Viscosity of Aceton-Water Solutions Up to Their Normal Builing Points. AIChe. E.J. 1958. Vol.4. No.3. P.362. 